H. Dai

K.T. Mu

J.P. Qi

C.Y. Wang

W.Z. Zhu

L.M. Xia

Z.Q. Chen

H. Zhang

F. Ai

J.N. Morelli

\section{ORIGINAL RESEARCH \\ Assessment of Lateral Geniculate Nucleus Atrophy with 3T MR Imaging and Correlation with Clinical Stage of Glaucoma}

BACKGROUND AND PURPOSE: Although previous animal studies have shown structural changes in ocular hypertension such as atrophy of the LGN, such changes have not been thoroughly studied in human glaucoma patients nor correlation made with clinical stage. Our aim was to investigate prospectively LGN atrophy in patients with POAG using 3T MR imaging and correlation with the clinical stage of disease.

MATERIALS AND METHODS: Twenty-six patients with known POAG and 26 age-matched healthy volunteers were included in this institutional review board-approved study. All subjects underwent imaging on a 3T MR imaging system with a PD and GM sequence. LGN height and volume were measured by 2 blinded neuroradiologists. Measurements were compared and correlated with clinical glaucoma severity as assessed by static threshold visual field parameters.

RESULTS: Average maximum LGN height in patients with glaucoma on PD images was $4.36 \pm 0.61$ $\mathrm{mm}$ (right) and $4.31 \pm 0.61 \mathrm{~mm}$ (left), significantly less $\left(P<10^{-3}\right)$ than respective measurements of $5.05 \pm 0.41$ and $4.99 \pm 0.41 \mathrm{~mm}$ in volunteers. With the $\mathrm{GM}$ sequences, such respective measurements were also less $\left(P<10^{-3}\right)$ in patients with glaucoma $(4.20 \pm 0.71 \mathrm{~mm}$ right, $4.00 \pm 0.85 \mathrm{~mm}$ left) versus respective measurements in volunteers $(4.88 \pm 0.51 \mathrm{~mm}$ right, $4.77 \pm 0.47 \mathrm{~mm}$ left). Average LGN volumes in the patient group were $98.0 \pm 27.2 \mathrm{~mm}^{3}$ (right) and $93.7 \pm 25.8 \mathrm{~mm}^{3}$ (left) with the PD sequence versus respective measurements of $85.2 \pm 27.1$ and $80.5 \pm 23.6 \mathrm{~mm}^{3}$ with the $\mathrm{GM}$ sequence. All height and volume measurements were greater in volunteers $\left(P<10^{-3}\right)$. In the patient group, both maximum height and volume of the LGN with both sequences were significantly correlated with cumulative clinical glaucoma stage $(P<.05)$.

CONCLUSIONS: MR imaging measurements of LGN height and volume are diminished in patients with glaucoma, with the extent of atrophy correlating to clinical stage, suggesting a novel imaging marker of disease severity.

\begin{abstract}
ABBREVIATIONS: BRAVO = brain volume imaging; $F A=$ fractional anisotropy; $f M R I=$ functional $M R$ imaging; $G M=$ gray matter; $G M h L=$ maximum height of the left $L G N$ with the $G M$ sequence; $\mathrm{GMhR}$ = maximum height of the right $\mathrm{LGN}$ with the $\mathrm{GM}$ sequence; GMvL = maximum volume of the left LGN with the GM sequence; GMvR = maximum volume of the right LGN with the GM sequence; $L G N=$ lateral geniculate nucleus; $M D=$ mean diffusivity; $M D S=$ mean deviation score; $\mathrm{PD}=$ proton density; PDhL = maximum height of the left $L G N$ with the PD sequence; PDhR = maximum height of the right $L G N$ with the PD sequence; $P D V L=$ volume of the left $L G N$ with the $P D$ sequence; $P D V R=$ volume of the right $L G N$ with the $P D$ sequence; $P O A G$ = primary open-angle glaucoma; stage $A=$ the sum of the clinical stages of both eyes; stage $L=$ the clinical stage of the left eye of a patient with POAG; stage $R=$ the clinical stage of the right eye of a patient with POAG; Sum $=$ the summation of height and volume bilaterally
\end{abstract}

G aucoma is a major cause of irreversible blindness worldwide, estimated to have affected 61 million people in 2010. ${ }^{1}$ Glaucoma is characterized by progressive degeneration of retinal ganglion cells and axons. ${ }^{2}$ Neurodegeneration associated with POAG is thus not restricted to the retinal ganglion cells and extends to the target neurons in the LGN. ${ }^{3,4}$ In POAG, a disease often characterized by elevated intraocular pressure, the primary site of damage appears to be the optic nerve in the region of the lamina cribrosa where the axons of

Received October 1, 2010; accepted after revision November 18.

From the Departments of Radiology (H.D., K.T.M., J.P.O., C.Y.W., W.Z.Z., L.M.X., F.A.) and Ophthalmology (Z.Q.C., H.Z.), Tongji Hospital, Huazhong University of Science and Technology University, Wuhan, China; and Department of Radiology (J.N.M.), Scott and White Memorial Hospital, Texas A\&M University, Temple, Texas.

Please address correspondence to Jianpin Qi, PhD, Department of Radiology, Tongji Hospital, Jiefang Rd 1095\#, Wuhan, China; e-mail: qijp2k01@yahoo.com

DOI 10.3174/ajnr.A2486 retinal ganglion cells form the optic nerve. This damage is thus likely a consequence of retrograde degeneration of ganglion cells within the retina itself. Moreover, in the visual system, as in other parts of the brain, neurons depend on connections with other neurons for proper function and survival. Consequently, the trans-synaptic changes within the LGN occur after the steady loss of the retinal ganglion cells and axons. ${ }^{4}$ In primates, the LGN is comprised of 6 distinct layers of neurons. As shown in a study by Ito, ${ }^{5}$ layers 1,4 , and 6 of the contralateral LGN and layers 2, 3, and 5 of the ipsilateral LGN receive input from the glaucomatous eye. It is thus supposed that the size of the LGN on a given side could be affected by glaucoma stages of both the left and right eyes.

Experimental studies in animals have examined LGN atrophy in glaucoma, though only 2 recent reports have demonstrated this finding in human patients with glaucoma. ${ }^{6,7}$ Gupta et $\mathrm{al}^{6}$ reported the first clinicopathologic case of human 


\section{Number of Eyes for Each Glaucoma Stage}
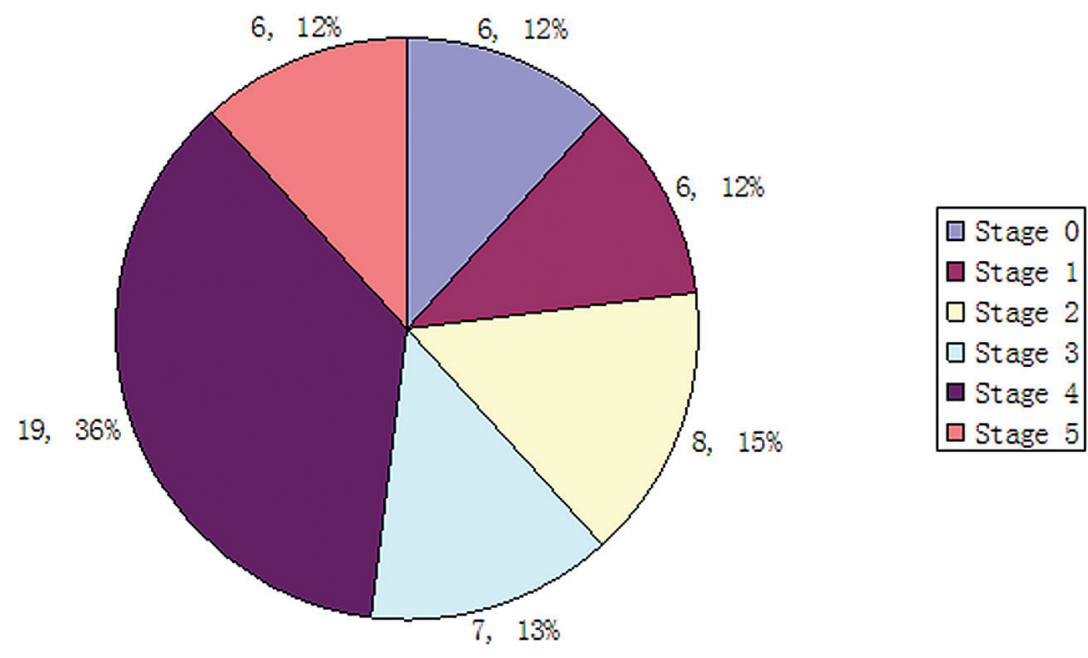

Fig 1. The pie chart shows the distribution of 52 eyes in 26 patients into different clinical stages.

glaucoma, demonstrating degenerative changes in the brain involving the intracranial optic nerves, LGN, and visual cortex in 2006. Three years later in vivo MR imaging evidence of LGN degeneration in human glaucoma was found, which was consistent with ex vivo primate and human neuropathologic studies. The aim of the present study was to further examine the MR imaging appearance of human glaucoma by correlating the stage of disease with the degree of LGN atrophy.

\section{Materials and Methods}

From March 2010 to August 2010, 2 groups of participants were included in this institutional review board-approved prospective study: a group of patients with glaucoma and a control group of healthy volunteers. Informed consent was obtained from all participants. Cardiovascular disease, diabetes, head trauma, and neurologic illness were excluded in both the POAG and control groups.

\section{Glaucoma Group and Classification}

The glaucoma group consisted of 26 patients with ages ranging from 21 to 58 years (mean, 35.4 years) with a diagnosis of POAG. This diagnosis was made on a clinical basis through assessment of the open anterior chamber angle, identification of visual field defects typical of glaucoma, optic disc cupping, or identification of an intraocular pressure of $\geq 21 \mathrm{~mm} \mathrm{Hg}$. In total, 21 male patients (range, 22-53 years of age; mean age, 34.4 years) and 5 female patients (range, $22-53$ years of age; mean age, 39.6 years) were included in the study.

The Hodapp-Anderson-Parrish system ${ }^{8}$ was used to stage the eyes of all patients with glaucoma (stages $0-5$ ). Stage 0 was characterized by increased intraocular pressure without evidence of visual field defects and with an MDS of $>0 \mathrm{~dB}$. Stage 1 (early) glaucoma was characterized by an MDS of -0.01 to $-6.00 \mathrm{~dB}$; stage 2 (moderate), by an MDS of -6.01 to $-12.00 \mathrm{~dB}$; and stage 3 (advanced) glaucoma, by an MDS of -12.01 to $-20.00 \mathrm{~dB}$. Stage 4 (severe) glaucoma was characterized by an MDS less than $-20.01 \mathrm{~dB}$. For patients designated clinically as having stage 5 , static threshold perimetry was not performed. A detailed accounting of eyes $(n=52)$ enrolled and their categorization by clinical stage is provided in the pie chart (Fig 1).

\section{Control Group}

The control group consisted of 26 healthy individuals with ages ranging from 21 to 57 years (mean, 35.4 years) who were matched by age and sex to the patient group. Twenty-one men (age range, 21-57 years; mean, 34.5 years) and 5 women (age range, $22-55$ years; mean, 38.6 years) were included as controls. All control subjects underwent an ophthalmologic examination via visual field testing to exclude glaucoma and other ocular diseases. There were no significant differences between the mean ages of the patient and control groups $(P=.8)$.

\section{MR Imaging}

All patients were scanned with a 3T MR imaging system (Signa HDxt; GE Healthcare, Milwaukee, Wisconsin). An 8-channel phased-array head coil was used for signal-intensity acquisition. Each subject was scanned head-first and supine. To reduce patient motion, the head position was fixed with foam cushions on both sides. The following sequences were acquired: an axial 3D BRAVO sequence (TR/TE/TI, $6.8 / 3.5 / 380 \mathrm{~ms}$; section thickness, $1 \mathrm{~mm}$ without intersection gaps; matrix, $256 \times 256$; FOV, $256 \mathrm{~mm}^{2}$; in-plane resolution, $1 \mathrm{~mm}$; NEX, 1; bandwidth, $42 \mathrm{~Hz}$; flip angle, $15^{\circ}$ ), an oblique coronal fast spinecho PD-weighted sequence (TR/TE, 3400/22 ms; section thickness, $1.8 \mathrm{~mm}$; matrix, $320 \times 320$; FOV, $256 \mathrm{~mm}^{2}$; in-plane resolution, 0.64 $\mathrm{mm}$; NEX, 4; bandwidth, $50 \mathrm{~Hz}$; echo-train length, 26), and an oblique coronal sequence with parameters designed for optimization of GM visualization (TR/TE/TI, 7600/24/300 ms; section thickness, $1.8 \mathrm{~mm}$ without intersection gaps; matrix, $320 \times 320$; FOV, $256 \mathrm{~mm}^{2}$; in-plane resolution, $0.64 \mathrm{~mm}$; NEX, 2; bandwidth, $15.63 \mathrm{~Hz}$; echotrain length, 12). The inversion pulses with the GM sequences were timed so as to reduce the signal intensity of white matter, thus theoretically improving visualization of the LGN. Both the PD and GM sequences were obtained with a homogeneous oblique coronal localizer, in a vertical orientation relative to the long axis of temporal lobe and medial hippocampus on a 3D BRAVO scan. This configuration is demonstrated in Fig 2. The 3D BRAVO sequence required only 2 minutes 40 seconds, much faster than the typically utilized 3D fastspoiled gradient recalled sequence. This sequence provided sufficient 


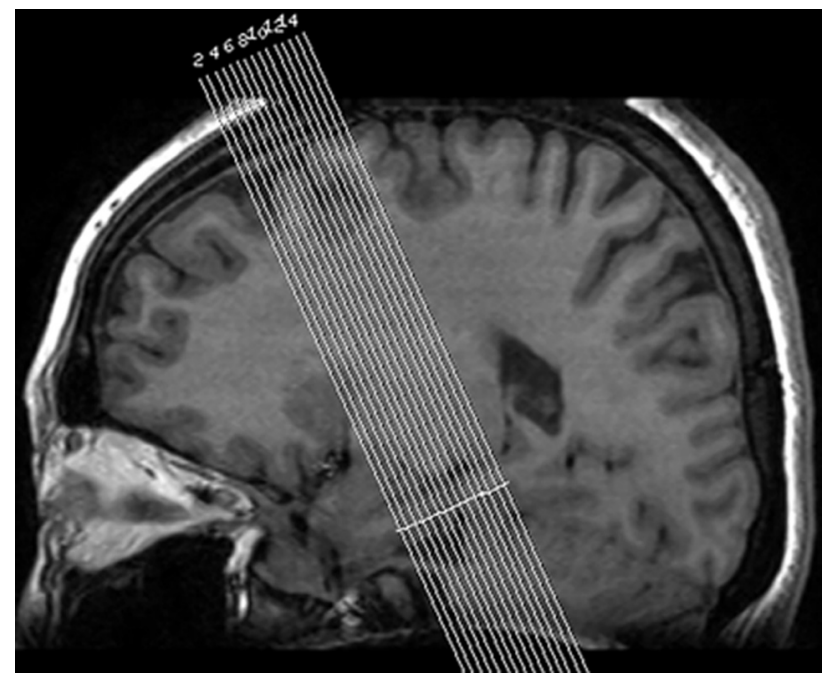

Fig 2. Oblique coronal localizer sequence demonstrates the orientation of acquired images for both the PD and GM sequences, perpendicular to the long axis of the temporal lobe or medial hippocampus.

spacial resolution, though less than the 3D fast-spoiled gradient recalled sequence, to serve as a 3D localizer for the PD and GM sequences.

\section{Measurement of the LGN}

The LGN is a small nucleus located at the lateral part of the metathalamus, with an internal geniculate nucleus inside. All measurements were made in consensus by 2 neuroradiologists who were blinded to the clinical diagnosis made by the ophthalmologists as detailed previously. Several stipulations on measurement technique were imposed on the readers: All images were magnified to an FOV of $90 \times 90$ $\mathrm{mm}^{2}$ before measurement, the vertical line in height measurements was drawn perpendicular to the base of LGN, and the oblique coronal planes on which the LGN was measured on PD and GM sequences were identical.

Image analyses were performed off-line on an Advantage workstation (AW 4.4; GE Healthcare, Buc, France). For each acquired sequence, LGN height measurements were performed by drawing a vertical line from the apex of the convexity to the base of the LGN as illustrated in Figs 3 and 4. The following measurements were obtained: the maximum PDhR, PDhL, GMhR, and GMhL. LGN volume measurements were performed by using a volume analysis tool available on the off-line workstation. On each scan section on which the LGN was visible, the area of the LGN was measured by using this tool as shown in Fig 5. These measurements were used to compute LGN volumes for the right LGN as obtained with the PDvR and PDvL, as well as the GMvR and GMvL.

All the height and volume measurements were corrected by the width of the temporal horn, an indirect measure of individual head size depicting the regional atrophy in the structures of the medial temporal lobe. ${ }^{9}$ Cumulative radius of the left and right temporal horn was calculated. The width of the temporal horn was measured on the GM sequence demonstrated in Fig 6.

\section{Statistical Analysis}

A general linear model was used to compare measurements of height and volume between the patient and control groups, by using the temporal horn width as a covariate to exclude the effect of head size and global atrophy on LGN size. The confidence intervals of multiple comparisons between patient and control groups were adjusted by the Bonferroni method. Statistical correlation between the height and volume versus the clinical stage of glaucoma was obtained for each eye and for each sequence via the Pearson nonparametric correlation test. The following parameters were also correlated: Sum versus stages L and $\mathrm{R}$ as well as stage A. All statistical analysis was performed with standard software (Statistical Package for the Social Sciences, Version 13.0; SPSS, Chicago, Illinois). A $P$ value $<.05$ was considered statistically significant for all comparisons.

\section{Results}

Subjectively, delineation of the LGN was much clearer on the GM sequence than on the PD images. This was due to both the improved gray-white matter differentiation with the former and the relatively higher signal intensity of CSF outlining the LGN and the Virchow-Robin spaces in the parenchyma adjoining the LGN. Representative images obtained from both sequences are provided in Fig 7.

Because the section thickness specified in both PD and GM sequences was $1.8 \mathrm{~mm}$, the LGN was typically visible in patients on 3 or 4 oblique coronal sections versus 4 sections in the volunteer group. As measured on the images obtained with the PD sequence, the mean value of the maximum height of the LGN in the patient group was $4.36 \pm 0.61 \mathrm{~mm}$ on the right and $4.31 \pm 0.61 \mathrm{~mm}$ on the left. The average measured LGN volume was $98.0 \pm 27.2 \mathrm{~mm}^{3}$ on the right and $93.7 \pm 25.8 \mathrm{~mm}^{3}$ on the left. For the GM sequence, the corresponding values were respectively $4.20 \pm 0.71 \mathrm{~mm}, 4.00 \pm 0.85 \mathrm{~mm}, 85.2 \pm$ $27.1 \mathrm{~mm}^{3}$, and $80.5 \pm 23.6 \mathrm{~mm}^{3}$. There were statistically significant differences between the patient and control groups with both sequences in terms of the maximum LGN height and volume after correction for individual head size $\left(P<10^{-3}, n=26\right)$. These differences are demonstrated in Table 1. Covariates appearing in the model were evaluated at the following value: cumulative temporal horn width = 4.3885. The tests between each variable of LGN size and temporal horn width had homogeneous results: $P>.05$.

The height and volume of both the right and left LGN as measured on PD and GM sequences were statistically significantly correlated with the cumulative stage of patient disease (stage A) though the $r$ value was not high, ranging from 0.455 to $0.613(P<.05, n=26)$. There was a correlation between stage L and bilateral LGN measurements from both sequences, with the exception of values for right LGN height obtained with the PD sequence $(P=.077)$. The volume of LGN measured with GM images and the height of the right LGN for both sequences were not correlated with the clinical glaucoma stage of the right eye (stage $\mathrm{R}, P>.05$ ) (Table 2).

\section{Discussion}

The association between glaucoma and LGN atrophy, while previously demonstrated in animal studies, ${ }^{10-14}$ has only recently been shown in humans. ${ }^{7}$ The present study elaborates on this initial observation in a much larger patient cohort and correlates the degree of LGN atrophy to the clinical stage of glaucoma present. This article is the first, to the knowledge of the authors, to show this association, which may have important applications for noninvasive assessment of glaucoma severity. This finding furthermore 

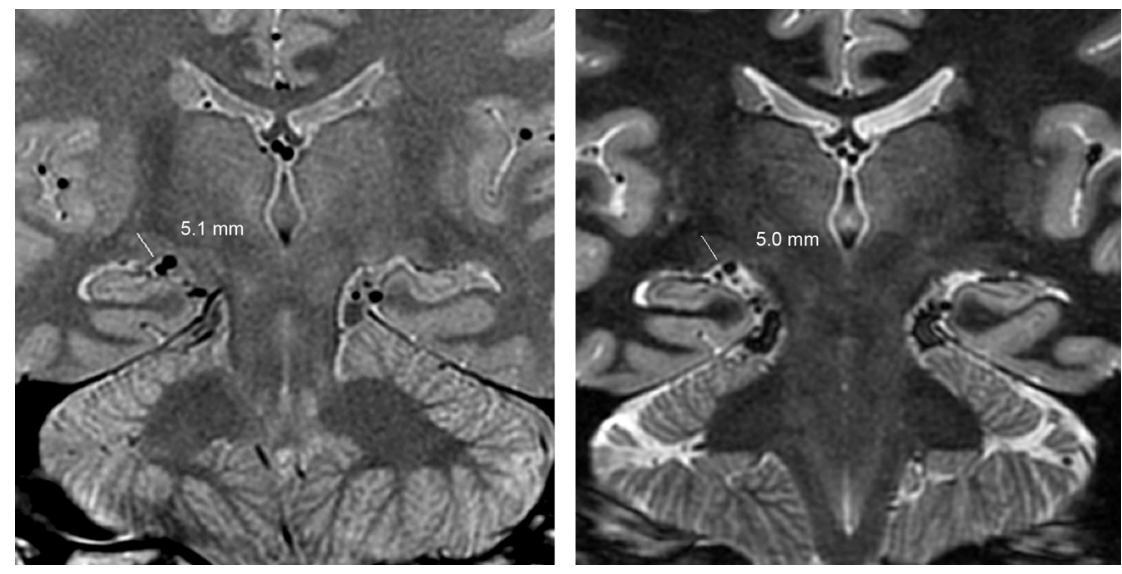

Fig 3. PD (left) and GM (right) images obtained in the identical oblique coronal plane demonstrate LGN height measurements in a 43-year-old healthy male volunteer. Specifically, LGN height is determined by drawing a line from the apex of the convexity perpendicular to the base of the LGN. The left image is from the PD sequence, and the right one, from GM sequence.
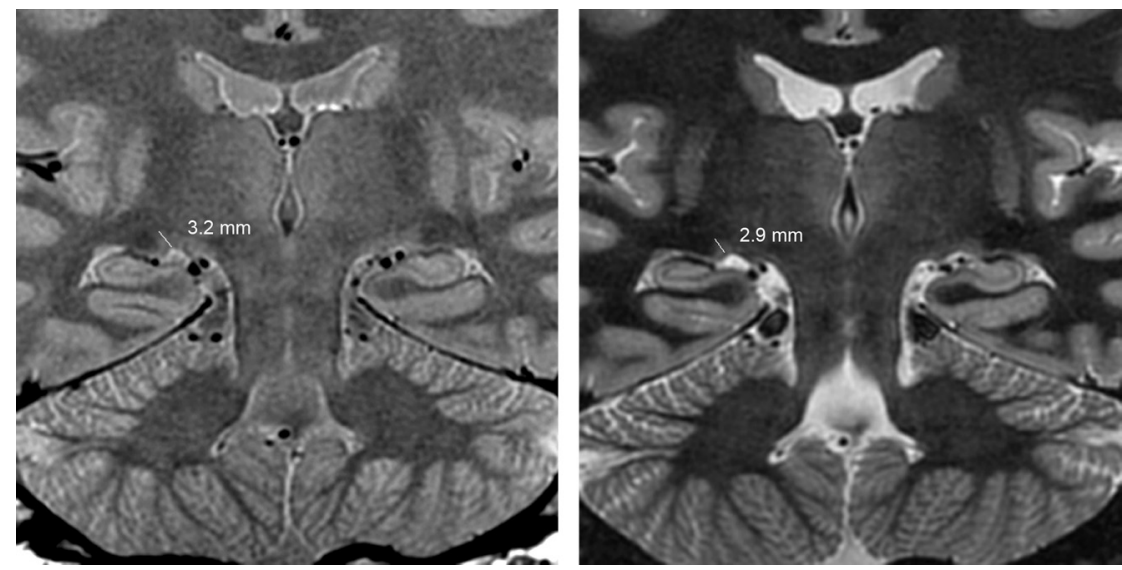

Fig 4. A 36-year-old man with bilateral stage 4 open-angle glaucoma demonstrates bilateral LGN atrophy as shown on both PD (left) and GM (right) sequences

suggests that early, as of yet clinically undetected, cases of glaucoma could be incidentally found on MR imaging examinations performed for other purposes, thus potentially facilitating earlier treatment.

This study is also the first to use LGN volume measurements in assessing LGN atrophy. Previous studies have suggested this as an alternative to measuring LGN height because it seems intuitive that a volume measurement accounting for LGN size in 3D would better correlate with glaucoma stage than would a $2 \mathrm{D}$ analysis. However, in the present study, it was found that both LGN height and volume correlate well with glaucoma stage. There is clinical value in this observation in that the maximum height measurement is, in practice, more readily obtained than the volume calculations described herein. Given the similar degree of correlation with glaucoma stage, measurements of LGN height would seem to be the preferred alternative for MR imaging assessment of glaucoma. Additionally in the present study, GM and PD-weighted sequences were compared with respect to the correlation of their LGN measurements with clinical stage. Subjectively, the GM images more clearly demarcated the LGN. Specifically, a key anatomic landmark, nearby CSF, was of high signal intensity on the GM sequence, thus improving LGN demarcation.

Suppression of signal intensity from white matter with the GM sequence very likely also improved the accuracy of quan- titative measurements due to less gray-white interface blurring; though measurements with both GM and PD sequences correlated equally well with glaucoma stage. Thus, while use of a dedicated GM sequence may aid in the qualitative assessment of the LGN in patients with glaucoma and may improve the precision of measurements obtained, as a practical matter, both PD and GM images do not appear to significantly differ in their correlation with glaucoma stage. Additional assessment of LGN atrophy detection and quantification with more commonly used MR imaging pulse sequences may be of further clinical value.

The interest in MR imaging assessment of glaucoma was spurred by pathologic studies at the level of the LGN and visual cortex, showing a smaller size of the former. These suggested a possible mechanism for neuroradiologic assessment of central visual pathways in glaucoma. ${ }^{15,16}$ Studies in the Japanese monkey glaucoma model ${ }^{5}$ further suggested that the magnocellular layer of the LGN may be more vulnerable than the parvocellular layer to the effects of elevated intraocular pressure. Specifically, this study found that layers 1,4 , and 6 of the contralateral LGN and layers 2, 3, and 5 of the ipsilateral LGN receive input from the glaucomatous eye. Additionally, optic chiasm, a well-known anatomic structure, over which the optic nerve crosses, strongly bears out the former finding. In other words, the size of each LGN is reflected by the clinical glaucoma stage of both eyes. 

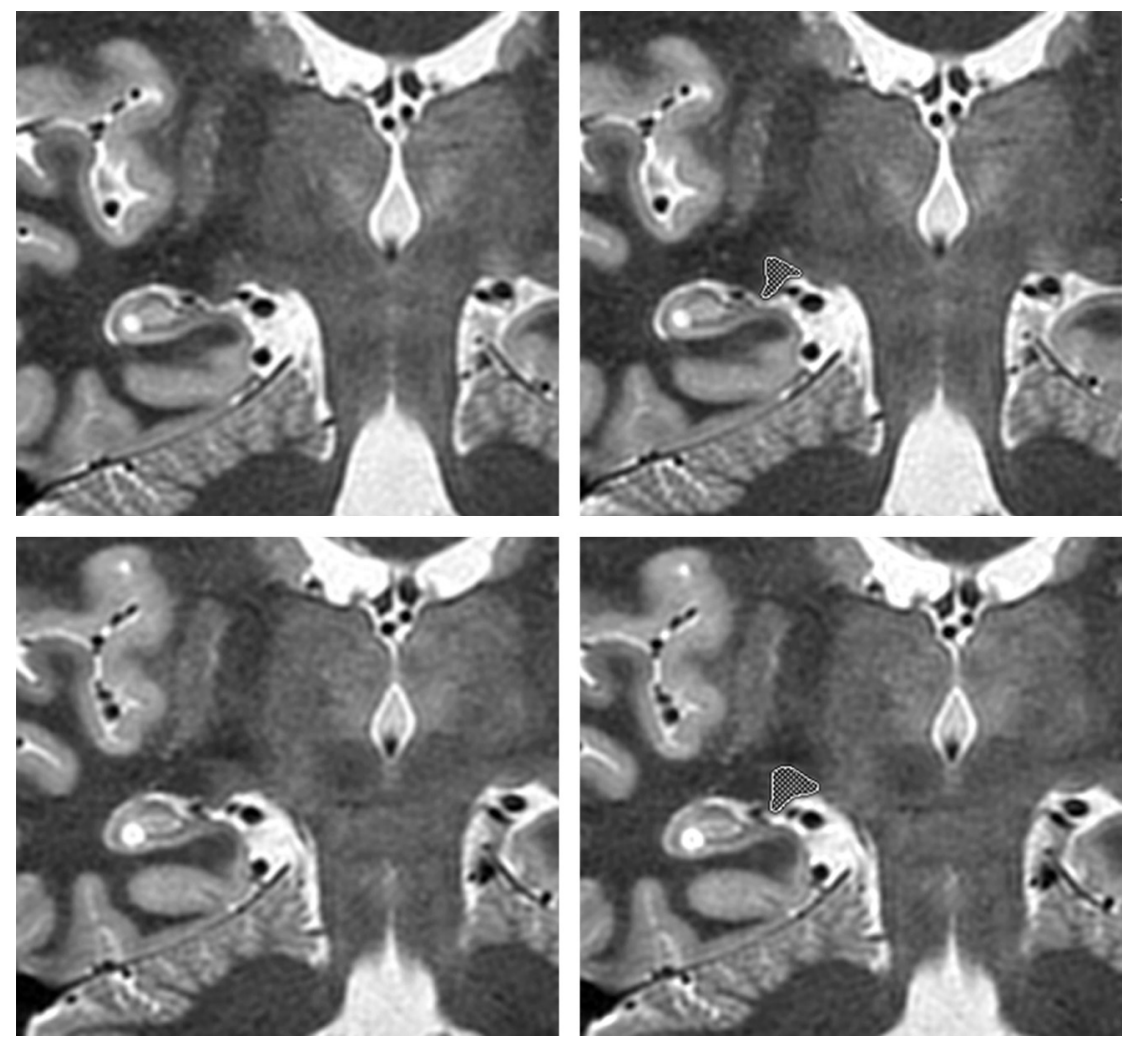

Fig 5. GM images demonstrate visualization of the LGN on 2 different oblique coronal sections in a healthy volunteer and show outlining of the LGN as performed for volume analysis.

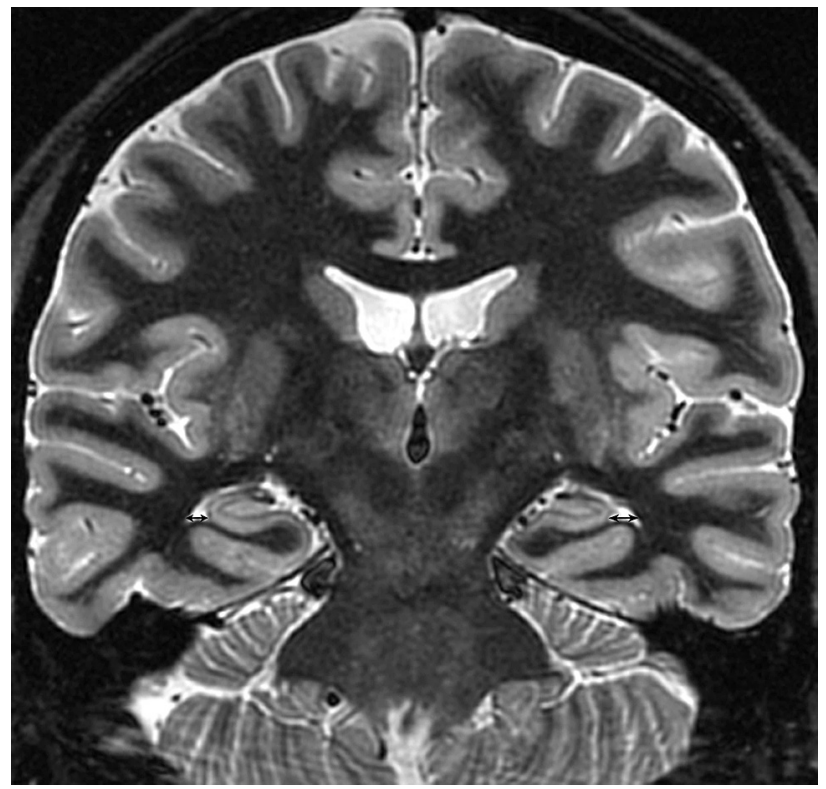

Fig 6. Temporal horns are measured on the GM sequence as a control brain region used to correct all the measurements of LGN size obtained from the PD and GM sequences.

Analysis of the variables "Sum" and "stage A" were included in the present study to further assess this relationship. On the basis of the data obtained, it appears that there is significant correlation between overall glaucoma stage (Stage A) and LGN size; however, as to stages L and R, there were several variables without significant correlation, though this may be confounded by several factors. For example, there were complex relations between the glaucoma stage in 1 eye versus the size in the contralateral or the ipsilateral LGN. Further study of this observation is thus warranted and may be better assessed in animal models, given the ability to readily obtain pathologic specimens.

MR imaging at 3T was initially applied to assess LGN atrophy in humans though several fMRI studies have evaluated changes in glaucoma. ${ }^{17,18}$ Garaci et $\mathrm{al}^{17}$ performed a diffusion tensor imaging scan on POAG patients, with MD and FA maps automatically created. They found that the optic radiations and optic nerves of patients with glaucoma, as compared with control subjects, had significantly higher MD and significantly lower FA. Further study showed the mean MD values for the optic nerves and the glaucoma stages varied consistently $(r=$ 0.8087, $P<.0001$ ) and there was a negative correlation between mean FA for the optic nerves and glaucoma stage $(r=$ $0.7464, P<.0001)$. Duncan et $\mathrm{al}^{18}$ found the spatial pattern of activity observed in the flattened representation agreed with the pattern of visual field loss and the amplitude of the blood oxygen level-dependent response was correlated on a pointwise basis with the difference in sensitivity thresholds between the glaucomatous and fellow eyes $(r=0.53, P<.0001)$. Additional applications of fMRI, which offer meaningful assessment of neural degeneration and functional alteration are the subject of further work by our laboratory. However, because POAG is an insidious disease, the time of diagnosis does not necessarily coincide with the time of disease onset, rendering disease chronicity difficult to establish. ${ }^{17}$ Furthermore, glaucoma that progresses at a variable rate, with fluctuations in intraocular pressure; the timeframe between staging of the glaucomatous disease and neuroimaging may thus not easily be established, especially in the early stage. 

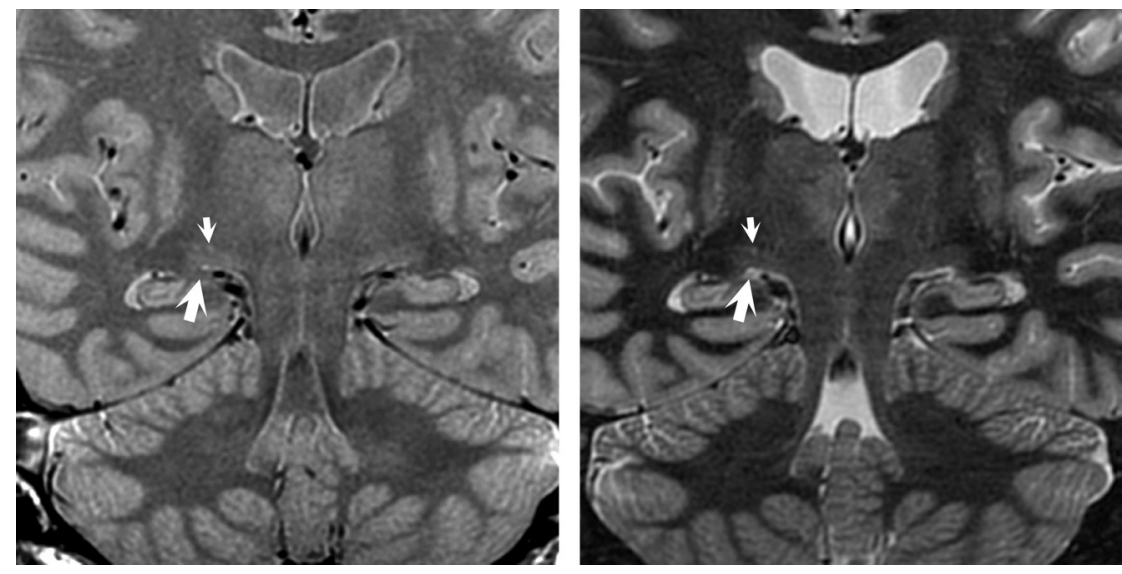

Fig 7. Clearer demarcation of gray and white matter is demonstrated in the GM sequence image (right), compared with the PD sequence image (left). Higher signal intensity of the Virchow-Robin space (small white arrows) and the CSF (large white arrows) adjacent to the LGN is notable on the GM sequences.

\begin{tabular}{|c|c|c|c|c|c|c|c|c|}
\hline & PDhR & PDhL & PDvR & PDvL & GMhR & GMhL & GMvR & GMvL \\
\hline Patients & $4.36 \pm 0.61$ & $4.31 \pm 0.61$ & $98.0 \pm 27.2$ & $93.7 \pm 25.8$ & $4.20 \pm 0.71$ & $4.00 \pm 0.85$ & $85.2 \pm 27.1$ & $80.5 \pm 23.6$ \\
\hline Controls & $5.05 \pm 0.41$ & $4.99 \pm 0.41$ & $143.5 \pm 22.3$ & $143.1 \pm 19.7$ & $4.88 \pm 0.51$ & $4.77 \pm 0.47$ & $131.7 \pm 18.5$ & $129.6 \pm 21.0$ \\
\hline
\end{tabular}

a Covariates appearing in the model are evaluated at the following value: temporal horn width (cumulative) $=4.3885$. ${ }^{\mathrm{b}}$ All $P$ values $<10^{-3}$

\begin{tabular}{|c|c|c|c|c|c|c|c|c|c|c|c|c|}
\hline Stage & PDhR & PDhL & Sum & PDvR & PDvL & Sum & GMhR & GMhL & Sum & GMvR & GMvL & Sum \\
\hline $\mathrm{L}$ & $\begin{array}{r}-.353^{\mathrm{a}, \mathrm{b}} \\
.077^{\mathrm{b}, \mathrm{c}}\end{array}$ & $\begin{array}{r}-.428 \\
.029\end{array}$ & $\begin{array}{r}-.405 \\
.040\end{array}$ & $\begin{array}{r}-.468 \\
.016\end{array}$ & $\begin{array}{r}-.525 \\
.006\end{array}$ & $\begin{array}{r}-.523 \\
.006\end{array}$ & $\begin{array}{r}-.400 \\
.043\end{array}$ & $\begin{array}{r}-.512 \\
.008\end{array}$ & $\begin{array}{r}-.473 \\
.015\end{array}$ & $\begin{array}{r}-.499 \\
.010\end{array}$ & $\begin{array}{r}-.478 \\
.014\end{array}$ & $\begin{array}{r}-.512 \\
.008\end{array}$ \\
\hline $\mathrm{R}$ & $\begin{array}{r}-.374^{b} \\
.060^{c}\end{array}$ & $\begin{array}{r}-.514 \\
.007\end{array}$ & $\begin{array}{r}-.461 \\
.018\end{array}$ & $\begin{array}{r}-.460 \\
.018\end{array}$ & $\begin{array}{r}-.401 \\
.042\end{array}$ & $\begin{array}{r}-.455 \\
.020\end{array}$ & $\begin{array}{r}-.330^{\mathrm{b}} \\
.099^{\mathrm{c}}\end{array}$ & $\begin{array}{r}-.395 \\
.046\end{array}$ & $\begin{array}{r}-.375^{\mathrm{b}} \\
.059^{\mathrm{c}}\end{array}$ & $\begin{array}{r}-.350^{\mathrm{b}} \\
.080^{\mathrm{c}}\end{array}$ & $\begin{array}{r}-.386^{b} \\
.093^{c}\end{array}$ & $\begin{array}{r}-.379^{\mathrm{b}} \\
.057^{\mathrm{c}}\end{array}$ \\
\hline A & $\begin{array}{r}-.455 \\
.019\end{array}$ & $\begin{array}{r}-.589 \\
.002\end{array}$ & $\begin{array}{r}-.541 \\
.004\end{array}$ & $\begin{array}{r}-.581 \\
.002\end{array}$ & $\begin{array}{r}-.582 \\
.002\end{array}$ & $\begin{array}{r}-.613 \\
.001\end{array}$ & $\begin{array}{r}-.458 \\
.019\end{array}$ & $\begin{array}{r}-.570 \\
.002\end{array}$ & $\begin{array}{r}-.533 \\
.005\end{array}$ & $\begin{array}{r}-.547 \\
.004\end{array}$ & $\begin{array}{r}-.520 \\
.006\end{array}$ & $\begin{array}{r}-.560 \\
.003\end{array}$ \\
\hline
\end{tabular}

$r$ value.

${ }^{b}$ Parameters without significant difference $(P>.05)$.

c $P$ value.

The present study has limitations. Although larger than previous studies assessing the relationship in LGN atrophy and glaucoma, the study population used is still relatively small. This may have, for example, affected the statistical power in determining whether volume or height measurements more closely correlated with degree of LGN atrophy. Furthermore, due to the fact that experimentation occurred in human patients, the results of LGN measurements could only be correlated with glaucoma staging rather than pathologic morphology. Although this correlation has been already examined extensively in rat and monkey models, providing the basis for the present study. As noted previously, only 2 of the many possible pulse sequences, the PD and GM sequences, on which LGN volume could be assessed were used in this model. Whether any other traditional fast spin-echo or gradient-echo T1- or T2-weighted images could be used in this field was not discussed. 2D volumetry by using a $1.8-\mathrm{mm}$ section thickness could demonstrate partial volume averaging because the target size with the averaging diameter was approximately $4.5 \mathrm{~mm}$. Additionally, voxel-based volumetry was not performed; the 3D BRAVO sequence acquired was substantially a 3D T1-weighted image, which did not clearly demarcate the LGN; thus no 3D segmentation was included. Patients with
POAG and volunteers were not matched or characterized on the basis of other factors that might potentially impact the right and left LGN size (other than glaucoma status), such as the presence of immune system disorders, race, sex, or handedness, though cardiovascular disease and diabetes were considered. Intraobserver and interobserver variation were also not tested in our study.

\section{Conclusions}

The present study confirms the loss in both LGN height and volume in patients with glaucoma and demonstrates that the stage of glaucoma correlates to degree of LGN atrophy. The latter fact suggests that a LGN examination by MR imaging may provide a useful noninvasive biomarker for the severity of glaucoma. Furthermore, evaluation of the visual pathways may be useful in the assessment of other degenerative conditions of the eye. However, further longitudinal studies with larger patient cohorts are needed to assess the accuracy of these parameters in determining glaucoma severity. MR imaging measurements of height and volume with the LGN are diminished in patients with glaucoma, with the extent of atrophy correlating to the clinical stage. These findings suggest a novel imaging marker of disease severity. 


\section{References}

1. Quigley HA, Broman AT. The number of people with glaucoma worldwide in 2010 and 2020. Br J Ophthalmol 2006;90:262-67

2. Weinreb RN, Khaw, PT. Primary open-angle glaucoma. Lancet 2004;363: $1711-20$

3. Luthra A, Gupta N, Kaufman PL, et al. Oxidative injury by peroxynitrite in neural and vascular tissue of the lateral geniculate nucleus in experimental glaucoma. Exp Eye Res 2005;80:43-49

4. Weber AJ, Chen H, Hubbard WC, et al. Experimental glaucoma and cell size, density, and number in the primate lateral geniculate nucleus. Invest Ophthalmol Vis Sci 2000;41:1370-79

5. Ito $\mathrm{Y}$, Shimazawa $\mathrm{M}$, Chen $\mathrm{YN}$, et al. Morphological changes in the visual pathway induced by experimental glaucoma in Japanese monkeys. Experimental Eye Research 2009;89:246-55.

6. Gupta N, Ang LC, de Tilly LN, et al. Human glaucoma and neural degeneration in intracranial optic nerve, lateral geniculate nucleus, and visual cortex. $\mathrm{Br} \mathrm{J}$ Ophthalmol 2006;90:674-78

7. Gupta N, Greenberg G, de Tilly LN, et al. Atrophy of the lateral geniculate nucleus in human glaucoma detected by magnetic resonance imaging. $\mathrm{Br} \mathrm{J}$ Ophthalmol 2009;93:56-60

8. Mills RP, Budenz DL, Lee PP, et al. Categorizing the stage of glaucoma from pre-diagnosis to end-stage disease. Am J Ophthalmol 2006;141:24-30

9. Frisoni GB, Beltramello A, Weiss C, et al. Linear measures of atrophy in mild Alzheimer disease. AJNR Am J Neuroradiol 1996;17:913-23

10. Yucel YH, Zhang Q, Gupta N, et al. Loss of neurons in magnocellular and parvocellular layers of the lateral geniculate nucleus in glaucoma. Arch Ophthalmol 2000;118:378-84

11. Yucel YH, Zhang Q, Weinreb RN, et al. Atrophy of relay neurons in magnoand parvocellular layers in the lateral geniculate nucleus in experimental glaucoma. Invest Ophthalmol Vis Sci 2001;42:3216-22

12. Yucel YH, Zhang Q, Weinreb RN, et al. Effects of retinal ganglion cell loss on magno-, parvo-, koniocellular pathways in the lateral geniculate nucleus and visual cortex in glaucoma. Prog Retin Eye Res 2003;22:465-81

13. Gupta N, Ly T, Zhang Q, et al. Chronic ocular hypertension induces dendrite pathology in the lateral geniculate nucleus of the brain. Exp Eye Res 2007;84: $176-84$

14. Zhang S, Wang H, Lu Q, et al. Detection of early neuron degeneration and accompanying glial responses in the visual pathway in a rat model of acute intraocular hypertension. Brain Res 2009;1303:131-43

15. Aldebasi YH, Drasdo N, Morgan JE, et al. Cortical OFF-potentials from the S-cone pathway reveal neural damage in early glaucoma. Vision Res 2003;43: 221-26

16. Sun $\mathrm{H}$, Swanson $\mathrm{WH}$, Arvidson $\mathrm{B}$, et al. Assessment of contrast gain signature in inferred magnocellular and parvocellular pathways in patients with glaucoma. Vision Res 2008;48:2633-41

17. Garaci FG, Bolacchi F, Cerulli A, et al. Optic nerve and optic radiation neurodegeneration in patients with glaucoma: in vivo analysis with 3-T diffusiontensor MR imaging. Radiology 2009;252:496-501

18. Duncan RO, Sample PA, Weinreb RN, et al. Retinotopic organization of primary visual cortex in glaucoma: comparing fMRI measurements of cortical function with visual field loss. Prog Retin Eye Res 2007;26:38-56 